\title{
Existence and Stability Property of Almost Periodic Solutions in Discrete Almost Periodic Systems
}

\author{
Yoshihiro Hamaya \\ Department of Information Science, Okayama University of Science 1-1 Ridai-cho, Okayama, Japan \\ Email: hamaya@icity.or.jp
}

How to cite this paper: Hamaya, Y. (2018) Existence and Stability Property of Almost Periodic Solutions in Discrete Almost Periodic Systems. Advances in Pure Mathematics, 8, 463-484.

https://doi.org/10.4236/apm.2018.85026

Received: February 26, 2018

Accepted: May 21, 2018

Published: May 24, 2018

Copyright (c) 2018 by authors and Scientific Research Publishing Inc. This work is licensed under the Creative Commons Attribution International License (CC BY 4.0).

http://creativecommons.org/licenses/by/4.0/

\section{(c) (i) Open Access}

\begin{abstract}
In this paper, we consider an almost periodic system which includes a system of the type $x(n+1)=\sum_{j-1}^{m} a_{i j}(n) x_{j}^{k}(1 \leq i \leq m)$, where $k$ is a positive integer, $a_{i j}$ are almost periodic in $n$ and satisfy $a_{i j}(n) \geq 0$ for $i \neq j, \quad \sum_{i=1}^{m} a_{i j}(n)=0$ for $1 \leq j \leq m$. In the special case where $a_{i j}(n)$ are constant functions, above system is a mathematical model of gas dynamics and was treated by $\mathrm{T}$. Carleman and R. D. Jenks for differential systems. In the main theorem, we show that if the $m \times m$ matrix $\left(a_{i j}(n)\right)$ is irreducible, then there exists a positive almost periodic solution which is unique and has some stability. Moreover, we can see that this result gives R. D. Jenks' result for differential model in the case where $a_{i j}(n)$ are constant functions. In Section 3, we consider the linear system with variable cofficients $x(n+1)=A(n) x(n), x \in R^{m}$. Even in nonlinear problems, this linear system plays an important role, as their variational equations, and it is requested to determine the uniform asymptotically stability of the zero solution from the information about $A(n)$. In order to obtain the existence of almost periodic solutions of both linear and nonlinear almost periodic discrete systems: above linear system and $x_{i}(n+1)=\sum_{j=1}^{m} a_{i j}(n) g_{j}\left(x_{j}(n)\right)$ for $1 \leq i \leq m$, respectively, we shall consider between certain stability properties, which are referred to as uniformly asymptotically stable, and the diagonal dominance matrix condition.
\end{abstract}

\section{Keywords}

Almost Periodic Solutions, Linear and Nonlinear Almost Periodic Discrete Systems, Uniformly Asymptotically Stable, Diagonal Dominance Matrix Condition 


\section{Introduction}

System of almost periodic difference equations has been studied to describe phenomena of oscillations in the natural and social sciences. The investigation of almost periodic systems has been developed quite widely during the twentieth century, since relationships with the stability theory have been found. A main interest of the subject is the existence theorem for almost periodic solutions. Obviously an almost periodic solution is a bounded solution, but the existence of bounded solutions does not necessarily imply the existence of almost periodic solutions. Therefore, in order to prove the existence of almost periodic solutions, we need some additional conditions to the existence of bounded solutions. A main subject of the investigation has been to find such additional conditions, and up to now, many conditions have been considered (for example, in the linear system, J. Favard's separation condition [1]).

In the Section 4, we consider the nonlinear almost periodic system of

$$
x_{i}(n+1)=\sum_{j=1}^{m} a_{i j}(n) x_{j}(n)^{k}, \quad 1 \leq i \leq m,
$$

where $k$ is a positive integer, $a_{i j}(n)$ are almost periodic in $n$ and satisfy

i) $\sum_{i=1}^{m} a_{i j}(n)=0,1 \leq j \leq m$,

ii) $a_{i j}(n) \geq 0$ for $i \neq j$.

In the special case where $a_{i j}(n)$ are constant functions, system (1) is a mathematical model of gas dynamics and was treated by T. Carleman [2] and R. D. Jenks [3]. In the main theorem, we show that if the $m \times m$ matrix $\left(a_{i j}(n)\right)$ is irreducible, then there exists a positive almost periodic solution which is unique and some stability. Moreover, we can see that this result gives R. D. Jenks' result in the case where $a_{i j}(n)$ are constant functions. In the Section 5, we consider the linear almost periodic system with variable coefficients

$$
x(n+1)=A(n) x(n), n \geq n_{0} \geq 0,
$$

where $x \in R^{m}$. Even in nonlinear problems, system (2) plays an important role, as their variational equations and moreover, it is requested to determine the uniformly asymptotic stability of the zero solution from the condition about $A(n)$. When $A(n)$ is a constant matrix, it is well known that the stability is equivalent to the following condition (cf. [4]);

"Absolute values of all eigenvalues of $A(n) \equiv A$ are less than one."

However, it is not true in the case of variable coefficients, and hence we need additional conditions to (2). In the main theorem, we show that one of the such conditions is the diagonal dominance matrix condition on $A(n)$ [5], that is, $A(n)$ satisfies

$$
\left|a_{i i}(n)\right| \geq \sum_{k=1, k \neq i}^{m}\left|a_{k i}(n)\right|, \quad 1 \leq i \leq m .
$$

This result improves a stability criterion based on results of F. Nakajima [6] for differential equations. 


\section{Preliminaries}

We denote by $R^{m}$ the real Euclidean $m$-space. Let $R=(-\infty, \infty)$ and $R^{+}=[0, \infty)$. $\mathrm{Z}$ is the set of integers, $\mathrm{Z}^{+}$is the set of nonnegative integers. For $x \in R^{m}$, let $|x|$ be the Euclidean norm of $x$ and $x_{i}$ be the $i$-th component. Let

$$
\begin{gathered}
D=\left\{x \in R^{m} \mid x_{i} \geq 0 \text { for } 1 \leq i \leq m\right\}, \\
\Omega=\left\{x \in D \mid \sum_{i=1}^{m} x_{i}=1\right\}
\end{gathered}
$$

and

$$
\Pi=\left\{x \in R^{m} \mid \sum_{i=1}^{m} x_{i}=0\right\} .
$$

We introduce an almost periodic function $f(n, x): \mathbf{Z} \times U \rightarrow R^{m}$, where $U$ is an open set in $R^{m}$.

Definition 1. $f(n, x)$ is said to be almost periodic in $n$ uniformly for $x \in U$, if for any $\epsilon>0$ and any compact set $K$ in $U$ there exists a positive integer $L^{*}(\epsilon, K)$ such that any interval of length $L^{*}(\epsilon, K)$ contains an integer $\tau$ for which

$$
|f(n+\tau, x)-f(n, x)| \leq \epsilon
$$

for all $n \in \mathbf{Z}$ and all $x \in K$. Such a number $\tau$ in above inequality is called an $\epsilon$-translation number of $f(n, x)$.

In order to formulate a property of almost periodic functions, which is equivalent to the above definition, we discuss the concept of the normality of almost periodic functions. Namely, let $f(n, x)$ be almost periodic in $n$ uniformly for $x \in U$. Then, for any sequence $\left\{h_{k}^{\prime}\right\} \subset \mathbf{Z}$, there exist a subsequence $\left\{h_{k}\right\}$ of $\left\{h_{k}^{\prime}\right\}$ and a function $g(n, x)$ such that

$$
f\left(n+h_{k}, x\right) \rightarrow g(n, x)
$$

uniformly on $\mathbf{Z} \times K$ as $k \rightarrow \infty$, where $K$ is a compact set in $U$. There are many properties of the discrete almost periodic functions [7], which are corresponding properties of the continuous almost periodic functions $f(t, x) \in C\left(R \times U, R^{m}\right)$ [cf. [8] [9]]. We denote by $T(f)$ the function space consisting of all translates of $f$, that is, $f_{\tau} \in T(f)$, where

$$
f_{\tau}(n, x)=f(n+\tau, x), \quad \tau \in \mathbf{Z} .
$$

Let $H(f)$ denote the uniform closure of $T(f)$ in the sense of (4). $H(f)$ is called the hull of $f$. In particular, we denote by $\Omega(f)$ the set of all limit functions $g \in H(f)$ such that for some sequence $\left\{n_{k}\right\}, n_{k} \rightarrow \infty$ as $k \rightarrow \infty$ and $f\left(n+n_{k}, x\right) \rightarrow g(n, x)$ uniformly on $\mathbf{Z} \times S$ for any compact subset $S$ in $R^{m}$. Specially, for a function $f(n)$ on $\mathbf{Z}$ with values in $R^{m}, H(f)$ denotes the set of all function $g(n)$ such that for some sequence $\left\{n_{k}\right\}$,

$$
f\left(n+n_{k}\right) \rightarrow g(n) \text { in } \mathbf{Z} \text { as } k \rightarrow \infty,
$$

where the symbol “ $\rightarrow$ ” stands for the uniformly convergence on any compact set 
in Z (in short, "in Z"). Clearly, $f \in H(f)$.

By (3), if $f: \mathbf{Z} \times U \rightarrow R^{m}$ is almost periodic in $n$ uniformly for $x \in U$, so is a function in $\Omega(f)$.

We define the irreducible matrix to need after.

Definition 2. An $m \times m$ matrix $A(n)=\left(a_{i j}(n)\right)$ is said to be irreducible if for any two nonempty disjoint subsets $I$ and $J$ of the set of $m$ integers $\{1,2, \cdots, m\}$ with $I \cup J=\{1,2, \cdots, m\}$, there exists an $i$ in $I$ and a $j$ in $J$ such that $a_{i j}(n) \neq \equiv 0$. In the case where $A(n)$ is scalar, $A(n)$ is said to be irreducible if $A(n) \not \equiv 0$. Otherwise, $A(n)$ is said to be reducible, and we can assume that $A(n)$ takes the form of

$$
A(n)=\left(\begin{array}{c|c}
* & 0 \\
\hline *^{\prime} & B(n)
\end{array}\right)
$$

where ${ }^{*}$ is $l \times l(1 \leq l \leq m)$ square matrix, ${ }^{*}$ is $m-l \times l$ matrix, $B(n)$ is $m-l \times m-l$ zero or a square irreducible matrix.

\section{Linear Systems}

We consider the system of linear difference equation

$$
x(n+1)=A(n) x(n),
$$

where $x \in R^{m}$ and the $m \times m$ matrix $A(n)=\left(a_{i j}(n)\right)$ is bounded on $\mathbf{Z}$ and almost periodic function in $n$. We state discretization of Jenks and Nakajima' results for differential equations [3] [10].

Now we define stability properties with respect to the subset $K$ in $R^{m}$. Here, we denote by $x\left(n, n_{0}, x_{0}\right)$ the solution of system (5) with initial condition $\left(n_{0}, x_{0}\right)$.

Definition 3. The bounded solution $u(n)$ of system (5) defined on $\mathbf{Z}$ is said to be;

i) uniformly stable (in short, U.S.) in $K$ on $\mathbf{Z}^{+}$if for any $\epsilon>0$ there exists a $\delta(\epsilon)>0$ such that $\left|u(n)-x\left(n, n_{0}, x_{0}\right)\right|<\epsilon$ for all $n \geq n_{0}$ whenever $x_{0} \in K$ and $\left|u\left(n_{0}\right)-x_{0}\right|<\delta(\epsilon)$ at some $n_{0}$ in $\mathbf{Z}^{+}$.

ii) uniformly asymptotically stable (in short, U.A.S.) in $K$ on $\mathbf{Z}^{+}$if it is U.S. in $K$ on $\mathrm{Z}^{+}$and if there exists a $\delta_{0}>0$ and, if for any $\epsilon>0$ there exists a $T(\epsilon)>0$ such that $\left|u(n)-x\left(n, n_{0}, x_{0}\right)\right|<\epsilon$ for all $n \geq n_{0}+T(\epsilon)$ whenever $x_{0} \in K$ and $\left|u\left(n_{0}\right)-x_{0}\right|<\delta_{0}$ at some $n_{0}$ in $\mathbf{Z}^{+}$.

iii) uniformly asymptotically stable (in short, U.A.S.) in the whole $K$ on $\mathbf{Z}^{+}$if it is U.S. in $K$ on $\mathbf{Z}^{+}$and if for any $\epsilon>0$ and $r>0$ there exists a $T(\epsilon, r)>0$ such that $\left|u(n)-x\left(n, n_{0}, x_{0}\right)\right|<\epsilon$ for all $n \geq n_{0}+T(\epsilon, r)$ whenever $x_{0} \in K$ and $\left|u\left(n_{0}\right)-x_{0}\right|<r$, at some $n_{0}$ in $\mathbf{Z}^{+}$.

When $\mathbf{Z}^{+}$in the definitions (i), (ii) and (iii) is replaced by $\mathbf{Z}$, we say that $x(n)$ is U.S. in $K$ on $\mathbf{Z}$, U.A.S. in $K$ on $\mathbf{Z}$ and U.A.S. in the whole $K$ on $\mathbf{Z}$, respectively. Clearly Definition 3 agrees with the definitions of the usual stability properties in the case where $K=R^{m}$.

Throughout this paper, we suppose the following conditions; 
i) $\sum_{i=1}^{m} a_{i j}(n)=0$ for $1 \leq j \leq m$,

ii) $a_{i j}(n) \geq 0$ for $i \neq j$

and

iii) each element in $H(A)$ is irreducible.

First of all, we prove the following lemmas.

Lemma 1. Consider the m-equations $x_{i}(n+1)=f_{i}(n, x(n)), 1 \leq i \leq m$, where $f_{i}(n, x)$ is continuous on second variable $x$ in $R^{m}$, and assume that the initial value problem has a unique solution.

a) If $\sum_{i=1}^{m} f_{i}(n, x)=0$, then the set $\Pi$ is invariant.

b) If $f_{i}(n, x) \geq 0$ for $x_{i}=0$ and $x_{j} \geq 0$, then the set $D$ is positively invariant, and in addition,

if $\sum_{i=1}^{m} f_{i}(n, x)=0$, then the set $\Omega$ is positively invariant.

In the case of differential equations, the proof of the similar lemma is obvious (for instance, see [11]). We modify it to prove this lemma, but we omit it.

Lemma 2. If conditions (i) and (ii) are satisfied, then the trivial solution of system (5) is U.S. in $\Pi$ on $\mathbf{Z}$ and also it is U.S. on $\mathbf{Z}$.

By modifying theorem in [5], we can easily prove Lemma 2 at same technique.

Lemma 3. If each element in $H(A)$ is irreducible, then the each element in $H(A)$, we say $B \in H(A)$ and $B(n)=\left(b_{i j}(n)\right)$, has the property that for any two nonempty disjoint subsets $I$ and $J$ of the set of $m$ integers $\{1,2, \cdots, m\}$ with $I \bigcup J=\{1,2, \cdots, m\}$, there exists an $i \in I$ and $j \in J$ such that

$$
\overline{\lim }_{n \rightarrow \infty}\left|b_{i j}(n)\right| \neq 0 \text {. }
$$

Proof. Suppose not, Then there exists a $B(n)=\left(b_{i j}(n)\right)$ in $H(A)$ and two nonempty disjoint subsets $I$ and $J$ of $\{1,2, \cdots, m\}$ with $I \cup J=\{1,2, \cdots, m\}$ such that

$$
\varlimsup_{n \rightarrow \infty} b_{i j}(n)=0 \text { for all } i \in I \text { and } j \in J .
$$

Since $B(n)$ is bounded on $\mathbf{Z}$, there exists a subsequence $n_{k}, n_{k} \rightarrow \infty$ as $k \rightarrow \infty$, such that

$$
B\left(n+n_{k}\right) \rightarrow C(n) \text { in } \mathbf{Z} \text { as } k \rightarrow \infty,
$$

where $C(n)=\left(c_{i j}(n)\right) \in H(A)$. Clearly,

$$
c_{i j}(n)=\lim _{k \rightarrow \infty} b_{i j}\left(n+n_{k}\right)=0 \text { for } n \in \mathbf{Z} \text { and } i \in I, j \in J .
$$

This show the reducibility of $C(n)$, which is a contradiction. This proves Lemma 3.

For system (5), we consider the system in $H(A)$ of

$$
x(n+1)=B(n) x(n)
$$

where $B \in H(A)$.

Lemma 4. Assume that conditions (ii) and (iii) are satisfied for system (5), 
and let $x(n)$ be a nontrivial solution of system (6) such that $x(n) \in D$ on $\mathbf{Z}$. Then there exists a constant $c>0$ such that

$$
x_{i}(n) /|x(n)| \geq c \text { for } n \in \mathbf{Z} \text { and } 1 \leq i \leq m .
$$

Proof. Let $x(n)=\left(x_{1}(n), x_{2}(n), \cdots, x_{m}(n)\right)$ be a solution of system (6) such that $x(n) \in D$ on $\mathbf{Z}$. First of all, we show that if $x_{i}\left(n_{0}\right)=0$ at some $n_{0} \in \mathbf{Z}$, then

$$
x_{i}(n)=0 \text { for all } n \geq n_{0}
$$

Since $x_{i}(n)$ satisfies the equation

$$
x_{i}(n+1)=b_{i i}(n) x_{i}(n)+\sum_{j \neq i}^{m} b_{i j}(n) x_{j}(n),
$$

where $\left(b_{i j}(n)\right)=B(n)$. Moreover, since $\sum_{j \neq i}^{m} b_{i j}(n) x_{j}(n) \geq 0$, we have

$$
x_{i}(n+1) \geq b_{i i}(n) x_{i}(n)
$$

which implies

$$
x_{i}(n) \leq x_{i}\left(n_{0}\right) \prod_{s=n_{0}}^{n-1} b_{i i}(s) \text { for } n \leq n_{0} \text {. }
$$

Thus, we obtain

$$
x_{i}(n)=0 \text { for } n \leq n_{0} .
$$

Because $x_{i}\left(n_{0}\right)=0$ and $x_{i}(n) \geq 0$ on $\mathrm{Z}$. Now suppose that Lemma 4 is not true. Then for some $B$ in $H(A)$, the corresponding system (6) has a nontrivial solution $x(n), x(n) \in D$ on $\mathbf{Z}$, such that for some sequence $n_{k}$,

$$
x_{1}\left(n_{k}\right) /\left|x\left(n_{k}\right)\right| \rightarrow 0 \text { as } k \rightarrow \infty .
$$

Set $\phi_{k}(n)=x\left(n+n_{k}\right) /\left|x\left(n_{k}\right)\right|$. Then, $\phi_{k}(n)$ satisfies

$$
x(n+1)=B\left(n+n_{k}\right) x(n)
$$

and

$$
\phi_{k}(n) \in D \text { on } \mathbf{Z},\left|\phi_{k}(0)\right|=1 \text {. }
$$

Since the sequence $\left\{\phi_{k}(0)\right\}$ is bounded, $\left\{\phi_{k}(n)\right\}$ is uniformly bounded on any finite interval in $\mathrm{Z}$, and hence there is a convergent subsequence of $\phi_{k}$, which is again denoted by $\phi_{k}$, such that

$\phi_{k}(n) \rightarrow y(n)$ in $\mathbf{Z}$ for some function $y(n)$ as $k \rightarrow \infty$.

We can also assume that

$$
B\left(n+n_{k}\right) \rightarrow C(n) \text { in } \mathbf{Z} \text { as } k \rightarrow \infty,
$$

where $C \in H(A)$ and $C(n)=\left(c_{i j}(n)\right)$. Therefore, $y(n)$ is the solution of the system

$$
y(n+1)=C(n) y(n)
$$

$y(n) \in D$ on $\mathbf{Z}$ and $|y(0)|=1$. Moreover (8) implies that $y_{1}(0)=0$. Thus, as was proved above, we have 


$$
y_{1}(n)=0 \text { for } n \leq 0,
$$

For this $y(n)$, we define two subsets $I$ and $J$ of $\{1,2, \cdots, m\}$ by $I=\left\{1 \leq i \leq m \mid y_{i}(n) \equiv 0\right.$ for $n \leq N_{i}$, where $N_{i}$ depends on $\left.y_{i}(n)\right\}$ and $J=\left\{1 \leq i \leq m \mid y_{i}(n)>0\right.$ on $\left.\mathbf{Z}\right\}$. Then $I \cup J=\{1,2, \cdots, m\}, \quad\{1\} \in I$ and $J \neq \phi$ since $y(n) \neq 0$. By Lemma 3 ,

$$
\varlimsup_{n \rightarrow \infty}\left|c_{i_{0} j_{0}}(n)\right| \neq 0 \text { for some } i_{0} \in I \text { and some } j_{0} \in J .
$$

Now the $i_{0}$-th equation of system (9) takes the form of

$$
y_{i_{0}}(n+1)=\sum_{k \in I} c_{i_{0} k}(n) y_{k}(n)+\sum_{k \in J} c_{i_{0} k}(n) y_{k}(n) \text {, }
$$

and hence

$$
\sum_{k \in J} c_{i_{0} k}(n) y_{k}(n)=0 \text { for } n+1 \leq \min _{i \in I} N_{i},
$$

because of the definition of the set $I$. Since each term in the left hand side of (11) is nonnegative, all of them are equal to zero. Therefore

$$
c_{i_{0} j_{0}}(n) y_{j_{0}}(n)=0 \text { for } n+1 \leq \min _{i \in I} N_{i},
$$

which implies, by (10),

$$
y_{j_{0}}\left(n_{0}\right)=0 \text { at some } n_{0} \text {. }
$$

This contradicts the definition of the set of $J$. The proof is completed.

The following proposition is an immediate result of Lemma 4.

Proposition 1. Under conditions (ii) and (iii), system (6) has no nontrivial solution $x(n)$ such that

$$
x(n) \in \partial D \text { on } \mathbf{Z},
$$

where $\partial D=\left\{x \in D \mid x_{i}=0\right.$ for some $\left.i, 1 \leq i \leq m\right\}$.

We next consider a non-homogeneous system corresponding to system (5)

$$
x(n+1)=A(n) x(n)+f(n)
$$

and assume that $A(n)$ satisfies conditions (i), (ii) and (iii).

Lemma 5. If $f(n)$ is bounded on $\mathbf{Z}$ with values in $R^{m}$ and $\sum_{i=1}^{m} f_{i}(n-1)$ is bounded on $\mathbf{Z}^{+}$, then all solutions of system (12) are bounded on $\mathbf{Z}^{+}$.

Proof. It is sufficient to show that (12) has at least one bounded solution on $\mathrm{Z}^{+}$, because the trivial solution of (5) is U.S. by Lemma 2 . We consider the system with real parameter $\epsilon$

$$
x(n+1)=A(n) x(n)+\epsilon f(n)
$$

and show that for a sufficiently small $\epsilon$, system (13) has a bounded solution on $\mathrm{Z}^{+}$, which implies the existence of a bounded solution on $\mathbf{Z}^{+}$for system (12) by replacing $x$ in (13) with $x / \epsilon$. For a $0<\delta<1 / \sqrt{m}$ and for the $m$-vector $e$ each of whose components is 1 , let $D^{\prime}$ be a convex cone defined by

$$
D^{\prime}=\left\{x \in R^{m}|\langle e, x\rangle \geq| e|\cdot| x \mid \cdot \delta\right\},
$$

where $\langle\cdot, \cdot\rangle$ denotes the inner product and $|x|^{2}=\langle x, x\rangle$. Clearly, $D \subset D^{\prime}$. 
Every solution $x(n)$ of (13) satisfies

$$
\sum_{i=1}^{m} x_{i}(n+1)=\epsilon \sum_{i=1}^{m} f_{i}(n)
$$

because of condition (i). By replacing $n$ with $n-1$ of the above both sides,

$$
\sum_{i=1}^{m} x_{i}(n)=\epsilon \sum_{i=1}^{m} f_{i}(n-1) \leq \tilde{M}+\epsilon M \text { for } x(0) \in \Omega,
$$

where

$\tilde{M}>0$ is sufficient small number and $M=\sup _{n>0} \sum_{i=1}^{m} f_{i}(n-1)$.

When $x(n) \in D^{\prime}$, we have

$$
\sum_{i=1}^{m} x_{i}(n)=\langle e, x(n)\rangle \geq|x(n)| \cdot|e| \cdot \delta,
$$

and hence,

$$
(\tilde{M}-\epsilon M) /|e| \leq|x(n)| \leq(\tilde{M}+\epsilon M) /(|e| \cdot \delta),
$$

Therefore, in order to show the boundedness of $x(n)$ with $x(0)$ in $\Omega$, it is sufficient to prove that $x(n) \in D^{\prime}$ on $\mathbf{Z}^{+}$if $\epsilon$ is sufficiently small. Now suppose that for each solution $x_{\epsilon}(n)$ of (13) with $x_{\epsilon}(0)$ in $\Omega$, there exists an $l_{\epsilon}>0$ such that

$$
x_{\epsilon}\left(l_{\epsilon}\right) \in \partial D^{\prime} .
$$

We can assume that

$$
x_{\epsilon}\left(n_{\epsilon}\right) \in \partial D \text { at some } n_{\epsilon}, 0 \leq n_{\epsilon}<l_{\epsilon}
$$

and

$$
x_{\epsilon}(n) \in \overline{D^{\prime}-D} \text { for } n_{\epsilon} \leq n \leq l_{\epsilon},
$$

where $\partial K$ and $\bar{K}$ denote the boundary and the closure of the set $K$, respectively. If we set $y_{\epsilon}(n)=x_{\epsilon}\left(n+n_{\epsilon}\right), y_{\epsilon}(n)$ is a solution of the system

$$
y(n+1)=A\left(n+n_{\epsilon}\right) y(n)+\epsilon f\left(n+n_{\epsilon}\right)
$$

such that $y_{\epsilon}(0) \in \partial D, y_{\epsilon}\left(\tau_{\epsilon}\right) \in \partial D^{\prime}$ at $\tau_{\epsilon}=l_{\epsilon}-n_{\epsilon}>0$ and $y_{\epsilon}(n) \in \overline{D^{\prime}-D}$ for $0 \leq n \leq \tau_{\epsilon}$. Thus, by (14),

$$
(\tilde{M}-\epsilon M) /|e| \leq\left|y_{\epsilon}(n)\right| \leq(\tilde{M}+\epsilon M) /(|e| \cdot \delta) \text { for } 0 \leq n \leq \tau_{\epsilon},
$$

The same argument in the proof of Lemma 4 enables us to assume that $y_{\epsilon}(n) \rightarrow w(n)$ in $\mathbf{Z}$ for some function $w(n)$ as $\epsilon \rightarrow 0$ and

$$
A\left(n+n_{\epsilon}\right) \rightarrow B(n) \text { in } \mathbf{Z} \text { for some } B \in H(A) \text { as } \epsilon \rightarrow 0
$$

Therefore, $w(n)$ satisfies $w(n+1)=B(n) w(n)$ and clearly, for $\tau=\liminf _{\epsilon \rightarrow 0} \tau_{\epsilon}$,

$$
w(n) \in \overline{D^{\prime}-D} \text { for } 0 \leq n<\tau
$$

and 


$$
\tilde{M} /|e| \leq|w(n)| \leq \tilde{M} /(|e| \cdot \delta) \text { for } 0 \leq n \leq \tau .
$$

Moreover we have $w(0) \in \partial D$, which implies by Lemma 1 that

$$
w(n) \in D \text { on } \mathbf{Z}^{+} \text {. }
$$

From this and (15) it follows that

$$
w(n) \in D \cap \overline{D^{\prime}-D}=\partial D \text { for } 0 \leq n<\tau .
$$

Now we show that $\tau=\infty$. In fact, if $\tau<\infty$, we have

$$
y_{\epsilon}\left(\tau_{\epsilon}\right) \rightarrow w(\tau) \text { as } \epsilon \rightarrow 0 .
$$

Thus $w(\tau) \in \partial D^{\prime}$ because $y_{\epsilon}\left(\tau_{\epsilon}\right) \in \partial D^{\prime}$, and hence

$$
w(\tau) \in \partial D^{\prime} \cap \partial D=\{0\},
$$

which contradicts (16). Therefore (16) and (17) hold for $\tau=\infty$. Moreover this enables us to assume that

$$
\tilde{M} /|e| \leq|w(n)| \leq \tilde{M} /(|e| \cdot \delta) \text { for all } n \in \mathbf{Z}
$$

and

$$
w(n) \in \partial D \text { for all } n \in \mathbf{Z} .
$$

Because $H(B)$ is compact in the sense of the convergence. This contradicts the conclusion in Proposition 1. This proves that $x(n) \in D^{\prime}$ on $\mathbf{Z}^{+}$if $\epsilon$ is sufficiently small. The proof is completed.

Lemma 6. Under the assumptions (i) and (ii), if for each $B$ in $H(A)$, the trivial solution of the system

$$
x(n+1)=B(n) x(n)
$$

is U.S. on $\mathbf{Z}$ and U.A.S. on $\mathbf{Z}^{+}$, then the trivial solution of system (5) is U.A.S. on $\mathbf{Z}$.

Proof. Let $x\left(n, n_{0}, x_{0}\right)$ be the solution of (5). Since the trivial solution of (5) is U.S. on $\mathbf{Z}$ by Lemma 2, as is seen from (ii) in Definition 3, it is sufficient to show that for any $\epsilon>0$ there exists a $T(\epsilon)>0$ such that

$$
\left|x\left(n, n_{0}, x_{0}\right)\right|<\delta(\epsilon) \text { for some } n, n_{0} \leq n \leq n_{0}+T(\epsilon),
$$

whenever $n_{0} \in \mathbf{Z}$ and $\left|x_{0}\right|<\delta_{0}=\delta(1)$, where $\delta(\cdot)$ is the number in (i) of Definition 3.

Now suppose that there exists an $\epsilon>0$ and sequences $\left\{n_{k}\right\}$ in $\mathbf{Z}$ and $\left\{x_{k}\right\}$ in $R^{m}$ such that $\left|x_{k}\right| \leq \delta_{0}$ and

$$
\left|x\left(n, n_{k}, x_{k}\right)\right| \geq \delta(\epsilon) \text { for all } n_{k}, n_{k} \leq n \leq n_{k}+k .
$$

Since $\left|x_{k}\right|<\delta_{0}=\delta(1)$,

$$
\delta(\epsilon) \leq\left|x\left(n, n_{k}, x_{k}\right)\right| \leq 1 \text { for } n_{k}, n_{k} \leq n \leq n_{k}+k .
$$

Set $\phi_{k}(n)=x\left(n+n_{k}, n_{k}, x_{k}\right)$. Then, $\phi_{k}(n)$ satisfies

$$
x(n+1)=A\left(n+n_{k}\right) x(n)
$$

and 


$$
\delta(\epsilon) \leq\left|\phi_{k}(n)\right| \leq 1 \text { for } 0 \leq n \leq k .
$$

We can assume that

$$
\phi_{k}(n) \rightarrow y(n) \text { in } \mathbf{Z}^{+} \text {for some function } y(n) \text { as } k \rightarrow \infty
$$

and

$A\left(n+n_{k}\right) \rightarrow B(n)$ in $\mathbf{Z}$ for some $B \in H(A)$ as $k \rightarrow \infty$.

Therefore $y(n)$ is a solution of the system

$$
y(n+1)=B(n) y(n)
$$

and

$$
\delta(\epsilon) \leq|y(n)| \leq 1 \text { on } \mathbf{Z}^{+} .
$$

On the other hand, we have

$$
y(n) \rightarrow 0 \text { as } n \rightarrow \infty,
$$

because the trivial solution of (18) is U.A.S. on $\mathrm{Z}^{+}$. Therefore there arises a contradiction. Thus the proof is completed.

We show the following theorem, before we will mention a definition of the exponential dichotomy of a linear system;

System (5) is said to possess an exponential dichotomy if there exists a projection matrix $P$ and positive constants $K_{1}, K_{2}, \rho_{1}, \rho_{2}, \sigma_{1}$ and $\sigma_{2}$ such that

$$
\begin{gathered}
\left\|\Phi(n) P \Phi^{-1}(l)\right\| \leq K_{1} \rho_{1}^{n-l}\left(0<\rho_{1}=\mathrm{e}^{-\sigma_{1}}\right), \text { for } n \geq l, \\
\left\|\Phi(n)(I-P) \Phi^{-1}(l)\right\| \leq K_{2} \rho_{2}^{n-l}\left(0<\rho_{2}=\mathrm{e}^{-\sigma_{2}}\right), \text { for } n \leq l,
\end{gathered}
$$

where, $I$ is a identical matrix and $\Phi$ is a fundamental matrix solution of system (5) (cf. [4] [5] [8]).

Theorem 1. Assume that system (5) satisfies conditions (i), (ii) and (iii), Then the trivial solution of system (5) is U.A.S. in $\Pi$ on $\mathbf{Z}$.

Proof. On the set $\Pi$ which is invariant for system (5), the system is written as the $(m-1)$-system

$$
\tilde{x}(n+1)=\tilde{A}(n) \tilde{x}(n)
$$

where $\tilde{x}=\left(x_{1}, x_{2}, \cdots, x_{m-1}\right) \in R^{m-1}$ and $\tilde{A}(n)$ is an $(m-1) \times(m-1)$ matrix whose $(i, j)$ element is given by $a_{i j}(n)-a_{i m}(n)$ for $1 \leq i, j \leq m-1$. First of all, we can show that for each $\tilde{B}$ in $H(\tilde{A})$, the system

$$
\tilde{x}(n+1)=\tilde{B}(n) \tilde{x}(n)
$$

has an exponential dichotomy on $\mathbf{Z}^{+}$since (20) has at least one bounded solution, and as is well known (cf. [12]), it is equivalent to show the system

$$
\tilde{x}(n+1)=\tilde{B}(n) \tilde{x}(n)+\tilde{f}(n)
$$

possesses at least one bounded solution on $\mathbf{Z}^{+}$for any bounded function $\tilde{f}(n)$ on $\mathbf{Z}^{+}$. For each $\tilde{B}(n)$ in $H(\tilde{A})$ there corresponds some $B(n)=\left(b_{i j}(n)\right)$ in $H(A)$ such that the $(i, j)$ element of $\tilde{B}(n)$ is equal to $b_{i j}(n)-b_{i m}(n)$ for $1 \leq i, j \leq m-1$. For $\tilde{f}(n)=\left(f_{1}(n), f_{2}(n), \cdots, f_{m-1}(n)\right)$, let $g(n)$ be defined by 


$$
\begin{gathered}
g_{i}(n)=f_{i}(n) \text { for } 1 \leq i \leq m-1, \\
g_{m}(n)=-\sum_{i=1}^{m-1} f_{i}(n) .
\end{gathered}
$$

Obviously $g(n)$ and $\sum_{i=1}^{m} g_{i}(n-1)(=0)$ are bounded on $\mathbf{Z}^{+}$. Applying Lemma 5 to the $m$-system

$$
x(n+1)=B(n) x(n)+g(n),
$$

we obtain the bounded solution $x(n)$ on $\mathrm{Z}^{+}$with $x(0)=0$, and $\sum_{i=1}^{m} g_{i}(n-1)=0 \quad$ which yields

$$
\sum_{i=1}^{m} x_{i}(n)=0 .
$$

Hence we can verify that $\tilde{x}(n)=\left(x_{1}(n), x_{2}(n), \cdots, x_{m-1}(n)\right)$ is a bounded solution on $\mathbf{Z}^{+}$of system (21). The exponential dichotomy of (20) implies further that the trivial solution is U.A.S. on $\mathbf{Z}^{+}$, because the trivial solution is U.S. on $\mathbf{Z}$ by Lemma 2. Therefore it follows from Lemma 6 that the trivial solution of (19) is U.A.S. on Z, i.e., the trivial solution of (5) is U.A.S. in $\Pi$ on $\mathbf{Z}$. The proof is completed.

\section{Nonlinear Systems}

We consider the nonlinear almost periodic system of

$$
x_{i}(n+1)=\sum_{j=1}^{m} a_{i j}(n) g_{j}\left(x_{j}(n)\right) \text { for } 1 \leq i \leq m,
$$

where $A(n)=\left(a_{i j}(n)\right)$ is almost periodic function of $n$ with conditions

(iv) $\sum_{i=1}^{m} a_{i j}(n)=0,1 \leq j \leq m$ and

(v) $a_{i j}(n) \geq 0$ for $i \neq j$.

In addition, assume that $g_{j}(u)$ are continuously differentiable for $u \geq 0$, $g_{j}(0)=0$ and $\dot{g}_{j}(u)>0$ for real number $u>0$, where $\dot{g}_{j}(u)$ is the derivative of $g_{j}(u)$ at $u$.

We first consider the linear system

$$
x(n+1)=L(n) x(n)
$$

and its perturbed system

$$
x(n+1)=L(n) x(n)+f(n, x(n)),
$$

where $L(n)$ is an $m \times m$ matrix function, almost periodic function in $n$, $f(n, x)$ is continuous with respect to its second argument and $f(n, x)=o(|x|)$ uniformly for $n \in \mathbf{Z}$. Assume that the set $\Pi$ is invariant for both system (23) and (24).

First of all, we can prove the following lemmas.

Lemma 7. If the trivial solution of system (23) is U.A.S. in $\Pi$ on $\mathbf{Z}$, then the trivial solution of system (24) has also the same stability property.

Proof. Let $\tilde{x}=\left(x_{1}, x_{2}, \cdots, x_{m-1}\right)$ for $x=\left(x_{1}, x_{2}, \cdots, x_{m}\right) \in R^{m}$. Then there are 
positive constants $c_{1}$ and $c_{2}$ such that

$$
c_{1}|\tilde{x}| \leq|x| \leq c_{2}|\tilde{x}| \text { for } x \in \Pi,
$$

because $x_{m}=-\left(x_{1}+x_{2}+\cdots+x_{m-1}\right)$. On the set $\Pi$, systems (23) and (24) are written as

$$
\tilde{x}(n+1)=\tilde{L}(n) \tilde{x}(n)
$$

and

$$
\tilde{x}(n+1)=\tilde{L}(n) \tilde{x}(n)+g(n, \tilde{x}(n))
$$

respectively, where the $(i, j)$ element of $\tilde{L}(n), 1 \leq i, j \leq m-1$, is given by $l_{i j}(n)-l_{i m}(n)$ for $L(n)=\left(l_{i j}(n)\right)$ and $g(n, \tilde{x})=o(|\tilde{x}|)$ uniformly for $n \in \mathbf{Z}$. Inequality (25) shows that the trivial solution of (23) is U.A.S. in $\Pi$ if and only if the trivial solution of (26) is U.A.S., and we have also the same equivalence between (24) and (27). As is well known, if the trivial solution of (26) is U.A.S., then the trivial solution of (27) has also the same stability property. Thus our assertion is proved.

The following lemma is obtained by the slight modification of the difference equation to Seifert's result [13]. Then, we will omit the proof (cf. [9]).

We consider the almost periodic nonlinear system

$$
x(n+1)=f(n, x(n)), n \in \mathbf{Z}, x \in R^{m},
$$

where $f(n, x)$ is almost periodic in $n$ uniformly for $x \in R^{m}$ and for a constant $L^{*}>0,|f(n, x)-f(n, y)| \leq L^{*}|x-y|$ for $n \in \mathbf{Z}$ and $x, y \in \Omega$.

Lemma 8. Assume that the set $\Omega$ is positively invariant for system (28) and all solutions in $\Omega$ on $\mathbf{Z}$ are U.A.S. in $\Omega$ on $\mathbf{Z}$. Then the set of such solutions is finite and consists of only almost periodic solutions $\phi_{1}, \phi_{2}, \cdots, \phi_{m}$ which satisfy

$\left|\phi_{i}(n)-\phi_{j}(n)\right| \geq \beta$ on $\mathbf{Z}$ for $i \neq j$ and some constant $\beta>0$.

Now we can show the following theorem. Since the last statements of the following theorem are alternative, under each assumption of these statements we can prove the existence of almost periodic solutions in $\Omega$ and the module containment.

Theorem 2. Under the assumptions (iv) and (v), system (22) has a nontrivial almost periodic solution in $\Omega$ whose module is contained in the module of $A(n)$. In addition to the above assumptions, if $A(n)$ is irreducible, then the almost periodic solution of (22) is unique in $\Omega$, which remains in $\Omega^{0}$ on $\mathbf{Z}$, and it is U.A.S. in the whole $\Omega$ on $\mathbf{Z}$, where $\Omega^{0}=\left\{x \in \Omega \mid x_{i}>0\right.$ for all $\left.i, 1 \leq i \leq m\right\}$. Moreover, if $A(n)$ is reducible, then at least one of the above almost periodic solutions $p(n)$ satisfies that $p(n) \in \partial \Omega$ on $\mathbf{Z}$, where $\partial \Omega=\left\{x \in \Omega \mid x_{i}=0\right.$ for some $\left.i, 1 \leq i \leq m\right\}$.

Proof. First of all, we consider the case where $A(n)$ is irreducible. Since system (22) satisfies the conditions of Lemma 1 , the set $\Omega$ is positively invariant, namely, $y(n) \in \Omega$ on $\mathbf{Z}^{+}$for a solution $y(n)$ of (22) with $y(0) \in \Omega$, and furthermore we can assume that 


$$
y(n) \in \Omega \text { on } \mathbf{Z}
$$

because of the almost periodicity of $A(n)$. We can show that this $y(n)$ is U.A.S. in $\Omega$ on Z. If we set $x=y(n)+w$ in system (22), then $w \in \Pi$ for $x$ in $\Omega$ and

$$
w_{i}(n+1)=\sum_{j=1}^{m} a_{i j}(n) \dot{g}_{j}\left(y_{j}(n)\right) w_{j}+o(|w|) \text { for } 1 \leq i \leq m,
$$

And $\Pi$ is invariant for the above system. Considering the first approximation of system (29)

$$
w(n+1)=L(n) w(n)
$$

where $L(n)=\left(l_{i j}(n)\right)$ is defined by $l_{i j}(n)=a_{i j}(n) \dot{g}_{j}\left(y_{j}(n)\right)$, condition (iv) implies that $\Pi$ is also invariant for (30). Then, by Lemma 6 , if the trivial solution of (30) is shown to be U.A.S. in $\Pi$ on Z, then the trivial solution of (29) has the same stability, and consequently $y(n)$ is U.A.S. in $\Omega$ on $\mathbf{Z}$. Therefore it is sufficient to show that the trivial solution of (30) is U.A.S. in $\Pi$ on Z. Clearly $L(n)$ is bounded and we have

$$
\sum_{i=1}^{m} l_{i j}(n)=\sum_{i=1}^{m} a_{i j}(n) \dot{g}_{j}\left(y_{j}(n)\right)=0 \text { for } 1 \leq j \leq m
$$

and

$$
l_{i j}(n)=a_{i j}(n) \dot{g}_{j}\left(y_{j}(n)\right) \geq 0 \text { for } i \neq j
$$

because of conditions (iv) and (v), respectively. Thus $L(n)$ satisfies conditions (i) and (ii). Condition (iii) will be verified in the following way. Applying the same argument as in the proof of Lemma 4 to system (22), we can see that there exists a constant $c>0$ such that

$$
1 \geq y_{i}(n) \geq c \text { for } n \in \mathbf{Z} \text { and } 1 \leq i \leq m,
$$

and hence there is a constant $\tilde{c}>0$ such that

$$
\dot{g}_{j}\left(y_{j}(n)\right) \geq \tilde{c} \text { for } n \in \mathbf{Z} \text { and } 1 \leq j \leq m .
$$

Therefore, (31) implies

$$
l_{i j}(n) \geq \tilde{c} a_{i j}(n) \text { for } i \neq j,
$$

which guarantees that each element of $H(L)$ is irreducible, because $A(n)$ is irreducible and almost periodic. Thus it follows from Theorem 1 that the trivial solution of (30) is U.A.S. in $\Pi$ on $\mathbf{Z}$, i.e., all solutions of system (22) in $\Omega$ on $\mathbf{Z}$ are U.A.S. in $\Omega$ on $Z$. Therefore Lemma 8 concludes that system (22) possesses an almost periodic solution in $\Omega$ which remains in $\Omega^{0}$ by (32), and the set of solutions in $\Omega$ on $\mathrm{Z}$ is finite and consists of only almost periodic solutions $\phi_{1}, \phi_{2}, \cdots, \phi_{m}$ which satisfy

$\left|\phi_{i}(n)-\phi_{j}(n)\right| \geq \beta$ on $\mathbf{Z}$ for $i \neq j$ and some constant $\beta>0$.

Next we can show that there exists a $T>0$ such that each solution $x\left(n, n_{0}, x_{0}\right)$ of (22) with $x_{0} \in \Omega$ satisfies that for some $\phi_{j}$ and the constant $\delta_{0}$ of (ii) in Definition 3, 


$$
\left|x\left(n, n_{0}, x_{0}\right)-\phi_{j}(n)\right|<\delta_{0} \text { at some } n, n_{0} \leq n \leq n_{0}+T
$$

which implies

$$
\left|x\left(n, n_{0}, x_{0}\right)-\phi_{j}(n)\right| \rightarrow 0 \text { as } n \rightarrow \infty,
$$

because $\phi_{j}$ is U.A.S. in $\Omega$. Suppose that this is not true. Then there exists a small constant $\alpha>0$ less than $\beta$ and sequences $\left\{n_{k}\right\}$ in $\mathbf{Z}$ and $\left\{x_{k}\right\}$ in $\Omega$ such that

$$
\left|x\left(n, n_{k}, x_{k}\right)-\phi_{j}(n)\right| \geq \alpha \text { for all } n \in\left[n_{k}, n_{k}+k\right] \text { and all } j, 1 \leq j \leq m .
$$

Since $f(n, x)$ is almost periodic in $n$ uniformly for $x \in \Omega$, we can choose a sequence $\left\{\tau_{k}\right\}, n_{k}+[k / 2]<\tau_{k}<n_{k}+k$, such that

$$
f\left(n+\tau_{k}, x\right) \rightarrow f(n, x) \text { in } \mathbf{Z} \times \Omega \text { as } k \rightarrow \infty .
$$

If we set $\psi_{j}^{(k)}(n)=\phi_{j}\left(n+\tau_{k}\right)$ for $1 \leq j \leq m$ and $\psi_{m+1}^{(k)}(n)=x\left(n+\tau_{k}, n_{k}, x_{k}\right)$, these $m+1$ functions $\psi_{j}^{(k)}(n)$ satisfy

$$
x(n+1)=f\left(n+\tau_{k}, x(n)\right)
$$

and

$$
\begin{gathered}
\psi_{j}^{(k)}(n) \in \Omega \text { on } \mathbf{Z} \text { for } 1 \leq j \leq m, \\
\psi_{m+1}^{(k)}(n) \in \Omega \text { for } n \geq-[k / 2] \geq n_{k}-\tau_{k},
\end{gathered}
$$

because $\psi_{m+1}^{(k)}\left(n_{k}-\tau_{k}\right)=x_{k} \in \Omega$. Moreover,

$$
\left|\psi_{j}^{(k)}(0)-\psi_{i}^{(k)}(0)\right| \geq \alpha \text { for } i \neq j, 1 \leq i, j \leq m+1 .
$$

We can assume that $\psi_{j}^{(k)}(n) \rightarrow \psi_{j}(n)$ in $\mathbf{Z}$ for some function $\psi_{j}, 1 \leq j \leq m+1$, as $k \rightarrow \infty$. Therefore $\psi_{j}(j=1,2, \cdots, m)$ are solutions of system (22), because $f\left(n+\tau_{k}, x\right) \rightarrow f(n, x)$ in $\mathbf{Z} \times \Omega$ as $k \rightarrow \infty$, and

$$
\psi_{j}(n) \in \Omega \text { on } \mathbf{Z} \text { for } 1 \leq i \leq m+1
$$

and

$$
\left|\psi_{j}(0)-\psi_{i}(0)\right|>\alpha \text { for } i \neq j, 1 \leq i, j \leq m+1,
$$

which shows that system (22) has $m+1$ distinct solutions in $\Omega$ on $\mathbf{Z}$. This is contradiction. Therefore, $\psi_{j}(n)$ is U.A.S. in the whole $\Omega$ on $\mathbf{Z}$, if the uniqueness of $\phi_{j}$ is shown.

Now we will prove the uniqueness of $\phi_{j}$. Suppose $\phi_{1} \neq \phi_{j}$ for $j \geq 2$ and set

$$
S_{1}=\left\{x_{0} \in \Omega|| x\left(n, 0, x_{0}\right)-\phi_{1}(n) \mid \rightarrow 0 \text { as } n \rightarrow \infty\right\}
$$

and

$$
S_{2}=\left\{x_{0} \in \Omega|| x\left(n, 0, x_{0}\right)-\phi_{j}(n) \mid \rightarrow 0 \text { for some } \phi_{j}, j \geq 2 \text {, as } n \rightarrow \infty\right\}
$$

Then $S_{1}$ and $S_{2}$ are open sets in $\Omega$, and moreover these sets are nonempty and disjoint, because $\left|\phi_{1}(n)-\phi_{j}(n)\right| \geq \alpha$ on $\mathbf{Z}$ for $j \geq 2$. On the other hand, (33) shows that $\Omega=S_{1} \cup S_{2}$, which contradicts the connectedness of $\Omega$. Thus 
the uniqueness of an almost periodic solution is proved, and moreover, as is seen from [14], this uniqueness guarantees the module containment of the almost periodic solution.

Now consider the case where $A(n)$ is reducible. We can assume $A(n)$ takes the form of

$$
A(n)=\left(\begin{array}{c|c}
* & 0 \\
\hline * & B(n)
\end{array}\right),
$$

where $B(n)$ is zero or a square irreducible matrix of order $l, 2 \leq l \leq m-1$. If $B(n)$ is zero, system (22) obviously has the constant solution $p(n)$ in $\partial \Omega$ such that $p_{i}(n)=0$ for $1 \leq i \leq m-1$ and $p_{m}(n)=1$. In the latter case, if we set in system (22)

$$
x_{k}=0 \text { for } 1 \leq k \leq l-m \text { and } y_{i}=x_{m-l+i} \text { for } 1 \leq i \leq l,
$$

then system (22) is reduced to the lower dimensional system

$$
y_{i}(n+1)=\sum_{j=1}^{l} b_{i j}(n) g_{j}\left(y_{j}(n)\right) \text { for } 1 \leq i \leq l,
$$

where $B(n)=\left(b_{i j}(n)\right)$. Since $B(n)$ is irreducible, the above system (34) has an almost periodic solution $y(n)$ such that

$$
y_{i}(n)>0 \text { for } 1 \leq i \leq l \text { and } \sum_{i=1}^{l} y_{i}(n)=1
$$

and furthermore the module of $y(n)$ is contained in the module of $B(n)$, i.e., of the module of $A(n)$. Thus, system (22) has an almost periodic solution $p(n)$ in $\partial \Omega$ on $\mathbf{Z}$ such that $p_{i}(n)=0$ for $1 \leq i \leq m-l$ and $p_{i}(n)=y_{i-m+l}(n)$ for $m-l+1 \leq i \leq m$. The proof is completed.

Remark 1. As will be seen from the module containment, the above almost periodic solution is a critical point in the case where $A(n)$ is a constant. Hence Theorem 2 is a discretization of Nakajimas' result (Theorem 2 in [10]).

\section{A Stability Criteria of Linear Systems}

We consider a stability criterion for solutions of a linear system with coefficient matrix of diagonal dominance type.

We again consider a linear system (5).

Let $A(n)=\left(a_{i j}(n)\right), i, j=1,2, \cdots, m$ be an $m \times m$ matrix of functions for $n \in \mathbf{Z}$. We assume the following conditions;

$$
\begin{gathered}
a_{j j}(n) \leq 0 \text { for } 1 \leq j \leq m \text { and } n \in \mathbf{Z}, \\
\operatorname{det} A(n) \neq 0,
\end{gathered}
$$

where, $\operatorname{det} A(n)$ denotes the determinant of matrix $A(n)$ and

$$
\sum_{i=1, i \neq j}^{m}\left|a_{i j}(n)\right| \leq\left|a_{i j}(n)\right| \text { for } 1 \leq j \leq m \text { and } n \in \mathbf{Z} .
$$

At first, we need the following lemmas for main theorem. 
Lemma 9. If a square matrix $A$ is irreducible and satisfies (38) and if for at least one $\mathrm{j}$,

$$
\sum_{i=1, i \neq j}^{m}\left|a_{i j}\right|<\left|a_{j j}\right|,
$$

then $A$ is nonsingular.

For the proof, see [15].

Lemma 10. If a nonsingular $m \times m$ matrix $A=\left(a_{i j}\right)$ satisfies (38), then all principal minors of $A$ are nonsingular, namely,

$$
\operatorname{det}\left(\begin{array}{cccc}
a_{j_{1} j_{1}} & a_{j_{1} j_{2}} & \cdots & a_{j_{1} j_{l}} \\
a_{j_{2} j_{1}} & a_{j_{2} j_{2}} & \cdots & a_{j_{2} j_{l}} \\
\vdots & \vdots & \ddots & \vdots \\
a_{j_{l} j_{1}} & a_{j_{l} j_{2}} & \cdots & a_{j_{l} j_{l}}
\end{array}\right) \neq 0 \text { for } 1 \leq j_{1}<j_{2}<\cdots<j_{l} \leq m \text {. }
$$

Proof. Let $A_{1}$ be an $l \times l(l<m)$ principal minor of $A$. Then, for a permutation matrix $Q$,

$$
Q A Q^{\mathrm{T}}=\left(\begin{array}{ll}
A_{1} & * \\
A_{2} & *
\end{array}\right),
$$

where $A_{2}$ has $(m-l)$ rows and $l$ columns and $Q^{\mathrm{T}}$ denotes the transposed matrix of $Q$. Moreover, from the definition of irreducibility, we can choose an $l \times l$ permutation matrix $Q_{l}$ such that

$$
Q_{l} A_{1} Q_{l}^{\mathrm{T}}=\left(\begin{array}{cccc}
B_{1} & C_{2} & & \\
& B_{2} & \ddots & \\
& & \ddots & C_{p} \\
& O & & B_{p}
\end{array}\right)
$$

where $B_{i}$ is an $r_{i} \times r_{i}$ irreducible matrix, $\sum_{i=1}^{p} r_{i}=l$, and $C_{i}$ has $\left(r_{1}+r_{2}+\cdots+r_{i-1}\right)$ row and $r_{i}$ columns for $2 \leq i \leq p$. In particular, in the case where $A_{1}$ is irreducible, $B_{1}$ must be $A_{1}$ itself, and the matrices $B_{2}, B_{3}, \cdots, B_{p}, C_{2}, C_{3}, \cdots, C_{p}$ are not present. Setting $P=\left(Q_{l} \oplus I\right) Q$ for $(m-l) \times(m-l)$ unit matrix $I$, where $Q_{l} \oplus I$ is the direct sum of $Q_{l}$ and $I$, we have

$$
B=P A P^{\mathrm{T}}=\left(\begin{array}{ccccc}
Q_{l} A_{1} Q_{l}^{\mathrm{T}} & * \\
A_{2} Q_{l}^{\mathrm{T}} & *
\end{array}\right)=\left(\begin{array}{ccccc}
B_{1} & C_{2} & & & C_{p} \\
& B_{2} & C_{3} & & C_{p} \\
& & \ddots & \ddots & \\
& & & B_{p-1} & C_{p} \\
& O & & & B_{p} \\
\hline D_{1} & D_{2} & & & D_{p}
\end{array}\right) *
$$

where $A_{2} Q_{l}^{\mathrm{T}}=\left(D_{1}, D_{2}, \cdots, D_{p}\right)$ and $D_{i}$ has $m-l$ rows and $r_{i}$ columns. Since the diagonal dominance condition (38) is invariant under the permutation of indexes, $B$ also satisfies (38). Hence, letting

$$
B_{i}=\left(b_{j k}\right), C_{i}=\left(c_{j k}\right) \text { and } D_{i}=\left(d_{j k}\right)
$$


for a fixed $i, 1 \leq i \leq p$, we have

$$
\sum_{j=1, j \neq k}^{r_{i}}\left|b_{j k}\right|+\sum_{j=1, j \neq k}^{r_{i}}\left|c_{j k}\right|+\sum_{j=1, j \neq k}^{r_{i}}\left|d_{j k}\right| \leq\left|b_{k k}\right| \quad\left(1 \leq k \leq r_{i}\right),
$$

where the summations on $j$ are taken along columns and $C_{1}=O$ for convenience. If $C_{i} \neq O$ or $D_{i} \neq O$, then

$$
\sum_{j=1, j \neq k}^{r_{i}}\left|c_{j k}\right|+\sum_{j=1, j \neq k}^{r_{i}}\left|d_{j k}\right|>0 \text { for some } k, 1 \leq k \leq r_{i},
$$

and hence for this $k$,

$$
\sum_{j=1, j \neq k}^{r_{i}}\left|b_{j k}\right|<\left|b_{k k}\right|
$$

by (39). Therefore it follows from Lemma 9 that

$$
\operatorname{det} B_{i} \neq 0 \text {, }
$$

since $B_{i}$ is irreducible. If $C_{i}=O$ and $D_{i}=O$, then we have the form of

$$
\operatorname{det} B=\operatorname{det} B_{i} \times(\cdots)
$$

which also implies (40), because $\operatorname{det} B \neq 0$. In any case, we have $\operatorname{det} B_{i} \neq 0$. Since these are true for all $i, 1 \leq i \leq p$, it follows from $\left(38_{1}\right)$ that

$$
\operatorname{det} A_{1} \neq 0 \text {. }
$$

this proves Lemma 10.

Lemma 11. If system (5) satisfies conditions (36) and (38), then the norm of solution $x(n)$ such that $|x(n)|=\sum_{i=1}^{m}\left|x_{i}(n)\right|$, is non-increasing, and consequently the zero solution is U.S..

For the proof, we can see (cf. [5]).

In the following theorem, we can prove that the zero solution is U.A.S., if $A(n)$ is bounded on $Z$ and if condition (36), (37) and (38) are satisfied.

Theorem 3. In system (5), let $A(n)$ be bounded on Z. Assume that conditions (36) and (38) are satisfied for all $n \in \mathbf{Z}$ and that there is a constant $\alpha>0$ such that

$$
|\operatorname{det} A(n)| \geq \alpha, \text { on } \mathbf{Z}
$$

Then the zero solution is U.A.S.

Proof. As is stated in Lemma 11, the zero solution is U.S., and hence it is sufficient to show that for any $\epsilon>0$ there exists a $T(\epsilon)>0$ such that

$$
\left|x\left(n, n_{0}, x_{0}\right)\right|<\epsilon \text { at some } n, n_{0} \leq n \leq n_{0}+T(\epsilon)
$$

whenever $\left|x_{0}\right| \leq 1$. Suppose that this is not true. Then there exists a constant $\epsilon>0$, a sequence of solution $\left\{x^{(k)}(n)\right\}$ of (5) and a sequence $n_{k}$ such that

$$
\left|x^{(k)}(n)\right| \geq \epsilon \text { on } n_{k} \leq n \leq n_{k}+k^{2}
$$

and

$$
\left|x^{(k)}\left(n_{k}\right)\right| \leq 1
$$


Since $\left|x^{(k)}(n)\right|$ is non-increasing, we have

$$
\epsilon \leq\left|x^{(k)}(n)\right| \leq 1 \text {, on } n_{k} \leq n \leq n_{k}+k^{2}
$$

and there exists a subinterval $\left[s_{k}, s_{k}+k\right]$ of $\left[n_{k}, n_{k}+k^{2}\right]$ such that

$$
|| x^{(k)}(n)|-| x^{(k)}\left(n^{\prime}\right)||<\frac{1}{k} \text {, for } s_{k} \leq n, n^{\prime} \leq s_{k}+k \text {. }
$$

Set $\varphi^{(k)}(n)=x^{(k)}\left(n+s_{k}\right)$. Then, we obtain

$$
\begin{gathered}
\varphi^{(k)}(n+1)=A\left(n+s_{k}\right) \varphi^{(k)}(n), \\
\epsilon \leq\left|\varphi^{(k)}(n)\right| \leq 1, \text { on } 0 \leq n \leq k
\end{gathered}
$$

and

$$
|| \varphi^{(k)}(n)|-| \varphi^{(k)}\left(n^{\prime}\right)||<\frac{1}{k}, \text { for } 0 \leq n, n^{\prime} \leq k .
$$

Since $A(n)$ is bounded, it follows from (41) and (42) that $\left\{\varphi^{(k)}(n)\right\}$ is uniformly bounded on any finite interval of $\mathbf{Z}$, and thus, taking a subsequence, $\left\{\varphi^{(k)}(n)\right\}$ can be assumed to converge uniformly on any finite interval of $\mathbf{Z}$. Defining $y(n)$ by

$$
y(n)=\lim _{k \rightarrow \infty} \varphi^{(k)}(n),
$$

it follows from (42) and (43) that there is a constant $\beta>0, \epsilon \leq \beta \leq 1$, such that

$$
|y(n)|=\beta \text {, for } n \geq 0 .
$$

Since $y(n)=\left(y_{1}(n), y_{2}(n), \cdots, y_{m}(n)\right)$ is defined on $\mathbf{Z}$, we can choose an interval $I=\left[\sigma_{1}, \sigma_{2}\right]$ (for some $\left.\sigma_{i} \in \mathbf{Z}(i=1,2), 0<\sigma_{1}<\sigma_{2}\right)$ such that

$$
\begin{gathered}
y_{j_{1}}(n), y_{j_{2}}(n), \cdots, y_{j_{h}}(n)>0 \text { on } I, \\
y_{j_{h+1}}(n), y_{j_{h+2}}(n), \cdots, y_{j_{l}}(n)<0 \text { on } I
\end{gathered}
$$

and

$$
y_{j_{l+1}}(n) \equiv y_{j_{l+2}}(n) \equiv \cdots \equiv y_{j_{m}}(n) \equiv 0 \text { on } I \text {. }
$$

Here we note that $\left\{j_{1}, j_{2}, \cdots, j_{l}\right\} \neq \phi$ because $y(n) \not \equiv 0$. Then

$$
|y(n)|=\sum_{p=1}^{h} y_{j_{p}}(n)-\sum_{p=h+1}^{l} y_{j_{p}}(n)=\beta \text { on } I .
$$

Let

$$
f^{(k)}(n)=\sum_{p=1}^{h} \varphi_{j_{p}}^{(k)}(n)-\sum_{p=h+1}^{l} \varphi_{j_{p}}^{(k)}(n) .
$$

Then, we have

$$
\lim _{k \rightarrow \infty} f^{(k)}(n)=|y(n)|=\beta \text { on } I,
$$

and there is a sequence $\left\{\theta_{k}\right\} \subset I$ such that

$$
\lim _{k \rightarrow \infty} \Delta f^{(k)}\left(\theta_{k}\right)=0 .
$$

Moreover, since $I$ is compact and $A(n)$ is bounded on Z, we can assume 
that

$$
\lim _{k \rightarrow \infty} \theta_{k}=\theta, \text { for some } \theta \in I
$$

and

$$
\lim _{k \rightarrow \infty} A\left(s_{k}+\theta_{k}\right)=B, \text { for some } m \times m \text { matrix } B .
$$

Clearly $B$ satisfies (36), (38) and $|\operatorname{det} B| \geq \alpha$. Taking a difference of both sides of (44) at $n=\theta_{k}$ and using relation (41), we find

$$
\Delta f^{(k)}\left(\theta_{k}\right)=\sum_{p=1}^{h}\left(\sum_{q=1}^{m} a_{j_{p} j_{q}}\left(s_{k}+\theta_{k}\right) \varphi_{j_{q}}^{(k)}\left(\theta_{k}, k\right)\right)-\sum_{p=h+1}^{l}\left(\sum_{q=1}^{m} a_{j_{p} j_{q}}\left(s_{k}+\theta_{k}\right) \varphi_{j_{q}}^{(k)}\left(\theta_{k}\right)\right),
$$

and

$$
\lim _{k \rightarrow \infty} \Delta f^{(k)}\left(\theta_{k}\right)=\sum_{p=1}^{h}\left(\sum_{q=1}^{l} b_{j_{p} j_{q}} y_{j_{q}}(\theta)\right)-\sum_{p=h+1}^{l}\left(\sum_{q=1}^{l} b_{j_{p} j_{q}} y_{j_{q}}(\theta)\right)
$$

where $B=\left(b_{i j}\right)$, since we have

$$
\lim _{k \rightarrow \infty} \varphi_{j}^{(k)}\left(\theta_{k}\right)=y_{j}(\theta), \quad \text { for } 1 \leq j \leq m
$$

and

$$
y_{j_{q}}(\theta)=0 \quad \text { for } l+1 \leq q \leq m .
$$

By (45), we have

$$
0=\sum_{q=1}^{l}\left(\sum_{p=1}^{h} b_{j_{p} j_{q}}-\sum_{p=h+1}^{l} b_{j_{p} j_{q}}\right) y_{j_{q}}(\theta) .
$$

Since $B$ satisfies (36) and (38),

$$
\sum_{p=1}^{h} b_{j_{p} j_{q}}-\sum_{p=h+1}^{l} b_{j_{p} j_{q}} \begin{cases}\leq 0, & \text { for } 1 \leq q \leq h, \\ \geq 0, & \text { for } h+1 \leq q \leq l .\end{cases}
$$

Therefore each term of right hand side of (46) is non-positive, because we have

$$
y_{j_{q}} \begin{cases}>0, & \text { for } 1 \leq q \leq h, \\ <0, & \text { for } h+1 \leq q \leq l\end{cases}
$$

Then it follows from (46) that

$$
\left(\sum_{p=1}^{h} b_{j_{p} j_{q}}-\sum_{p=h+1}^{l} b_{j_{p} j_{q}}\right) y_{j_{q}}(\theta)=0, \text { for } 1 \leq q \leq l,
$$

which implies

$$
\sum_{p=1}^{h} b_{j_{p} j_{q}}-\sum_{p=h+1}^{l} b_{j_{p} j_{q}}=0, \text { for } 1 \leq q \leq l,
$$

since $\left|y_{j_{q}}(\theta)\right| \neq 0$ for $1 \leq q \leq l$. Thus we have

$$
\operatorname{det}\left(\begin{array}{cccc}
b_{j_{1} j_{1}} & b_{j_{1} j_{2}} & \cdots & b_{j_{1} j_{l}} \\
b_{j_{2} j_{1}} & b_{j_{2} j_{2}} & \cdots & b_{j_{2} j_{l}} \\
\vdots & \vdots & \ddots & \vdots \\
b_{j_{l} j_{1}} & b_{j_{l} j_{2}} & \cdots & b_{j_{l} j_{l}}
\end{array}\right)=0
$$


On the other hand, $B$ satisfies (38) and $|\operatorname{det} B| \geq \alpha>0$, and thus it follows from Lemma 10 that all principal minors of $B$ are nonsingular, which contradicts $\left(46_{1}\right)$. This proves that the zero solution of system (5) is U.A.S..

Corollary 1. If system (5) is defined only for $n \geq 0$ and all assumptions of Theorem 3 are satisfied for $n \geq 0$, then the zero solution is U.A.S. for $n \geq 0$.

Proof. We construct the system defined on $\mathrm{Z}$ by

$$
x(n+1)=A_{0}(n) x(n), \quad x \in R^{m},
$$

where

$$
A_{0}(n)= \begin{cases}A(n), & \text { for } n \geq 0, \\ A(0), & \text { for } n<0 .\end{cases}
$$

Since system (47) satisfies all assumptions of Theorem 3 on $\mathbf{Z}$, the zero solution is U.A.S. on Z $\mathbf{Z}$, and furthermore, since system (5) coincides with system (47) for $n \geq 0$, this prove our conclusion.

\section{Application}

Before Example 1, we state the following lemma is a special case of Theorem 3 in [13].

In the nonlinear system

$$
x(n+1)=F(n, x(n)),
$$

let $F(n, x)$ be almost periodic in $n$ uniformly for $x \in R^{m}$ and for any $r>0$, let there exists a constant $L_{*}=L_{*}(r)>0$ such that

$$
|F(n, x)-F(n, y)| \leq L_{*}|x-y| \text { for }|x|,|y| \leq r \text { and } n \in \mathbf{Z} \text {. }
$$

Lemma 12. If $x(n)$ is a bounded solution of (48) on $\mathbf{Z}$ and if for any solution $y(n)$ of $(48),|x(n)-y(n)|$ is monotone decreasing to zero as $n \rightarrow \infty$, then $x(n)$ is a unique almost periodic solution and its module is contained in the module of $F(n, x)$.

Example 1. Consider the variational linear difference equation

$$
w(n+1)=A(n) w(n),
$$

where

$$
A(n)=\left(\begin{array}{cc}
-p(n) & \frac{\alpha}{2} \\
p(n)-\frac{2}{\alpha} q(n) & -\frac{\alpha}{2}
\end{array}\right) .
$$

We now assume that $\left(u_{0}(n), v_{0}(n)\right)$ is at least one bounded solution of (49) on $\mathbf{Z}$ and $(u(n), v(n))$ is any solution of (49) on $\mathbf{Z}$, and $p(n), q(n)$ are some bounded functions on $\mathbf{Z}$ such that

$$
p(n) \geq \alpha / 2 \text { and } \beta_{1} \leq q(n) \leq \beta_{2},
$$

for some positive constants $\alpha, \beta_{1}$ and $\beta_{2}$ such that $\alpha^{2} \geq 2 \beta_{2}$. We can verify that $A(n)$ satisfies all assumptions in Corollary 1. First of all, $A(n)$ is 
bounded in the future for $n \in \mathbf{Z}$, because $p(n)$ and $q(n)$ are bounded function on $\mathbf{Z}$. It is clear that the diagonal elements of $A(n)$ are negative and

$$
\operatorname{det} A(n)=q(n) \geq \beta_{1} \text { on } \mathbf{Z} .
$$

The diagonal dominance condition (38) for $A(n)$ requires that

$$
-p(n)+\left|p(n)-\frac{2}{\alpha} q(n)\right| \leq 0 \text {, and }-\frac{\alpha}{2}+\left|-\frac{\alpha}{2}\right| \leq 0,
$$

which is equivalent to

$$
q(n) \leq \alpha p(n)
$$

and this is satisfied by

$$
q(n) \leq \beta_{2} \leq \alpha^{2} / 2 \leq \alpha p(n) .
$$

Therefore, by Theorem 3, the zero solution of (49) is U.A.S. and

$$
\left|u_{0}(n)-u(n)\right|+\left|v_{0}(n)-v(n)\right| \rightarrow 0 \text { as } n \rightarrow \infty,
$$

where the convergence is monotone decreasing by Lemma 11 . Thus, applying Lemma 12 to system (49), we find that there exists a unique almost periodic solution with the module contained in the module of $A(n)$.

\section{Conclusion}

In this paper, we obtain the existence and stability property of almost periodic solutions in discrete almost periodic systems. First, in Section 1, the research background is introduced. In Section 2, the fundamental concepts of the almost periodic solutions in discrete almost periodic systems is given. In Section 3, we are introduced to the several lemmas and have uniformly asymptotically stability theory of the linear system, and moreover, in Section 4, we consider the generalized gas almost periodic system, and if linear part is irreducible matrix, then we obtain the existence of almost periodic solutions of this system. Finally, in Sections 5 and 6, we consider and obtain an uniformly asymptotically stability criterion for solutions of a linear system with coefficient matrix of diagonal dominance conditions, and this result applies to meaningful example of a linear discrete system.

\section{References}

[1] Favard, J. (1933) Lecons les functions presque-periodiques. Gauthier-Villars, Paris.

[2] Carleman, T. (1957) Problems Mathematiques dans la Theorie Cinetique des Gaz. Publ. Sci. Inst. Mittag-Leffler.

[3] Jenks, R.D. (1968) Homogeneous Multidimensional Differential Systems for Mathematical Models. Journal of Differential Equations, 4, 549-565. https://doi.org/10.1016/0022-0396(68)90005-3

[4] Elaydi, S. (2005) An Introduction to Difference Equations. 3rd Edition, Springer, Berlin.

[5] Coppel, W.A. (1965) Stability and Asymptotic Behavior of Differential Equations. Heath Mathematical Monographs, Heath, Boston. 
[6] Nakajima, F. (1978) A Stability Criterion of Diagonal Dominance Type. SIAM Journal on Mathematical Analysis, 9, 815-824. https://doi.org/10.1137/0509063

[7] Corduneanu, C. (1982) Almost Periodic Discrete Processes. Libertas Mathematica, 2, 159-169.

[8] Fink, A.M. (1974) Almost Periodic Differential Equations. Lecture Notes in Mathematics 377, Springer-Verlag, Berlin.

[9] Yoshizawa, T. (1975) Stability Theory and the Existence of Periodic Solutions and Almost Periodic Solutions. Applied Mathematical Sciences 14, Springer-Verlag, Berlin. https://doi.org/10.1007/978-1-4612-6376-0

[10] Nakajima, F. (1976) Existence and Stability of Almost Periodic Solutions in Almost Periodic Systems. Publications of the Research Institute for Mathematical Sciences, Kyoto Univ., Kyoto, 12, 31-47. https://doi.org/10.2977/prims/1195190957

[11] Krasnoselskii, M.A. (1964) Positive Solutions of Operator Equations. P. Noordhoff Ltd., Gronigen.

[12] Massera, J.L. and Schaffer, J.J. (1958) Linear Differential Equations and Functional Analysis. Annals of Mathematics, 67, 517-573. https://doi.org/10.2307/1969871

[13] Seifert, G. (1968) Almost Periodic Solutions and Asymptotic Stability. Journal of Mathematical Analysis and Applications, 21, 136-149. https://doi.org/10.1016/0022-247X(68)90248-5

[14] Montandon, B. (1972) Almost Periodic Solutions and Integral Manifolds for Weakly Nonlinear Nonconservative Systems. Journal of Differential Equations, 12, 417-425. https://doi.org/10.1016/0022-0396(72)90015-0

[15] Varga, R.S. (1962) Matrix Iterative Analysis. Prentice-Hall, Englewood Cliffs. 